\title{
Population Receptive Field Shapes in Early Visual Cortex Are Nearly Circular
}

\author{
${ }^{\circledR}$ Garikoitz Lerma-Usabiaga, ${ }^{1,2,3}{ }^{\circledR}$ Jonathan Winawer, ${ }^{4}$ and ${ }^{\circledR}$ Brian A. Wandell ${ }^{1,2}$ \\ ${ }^{1}$ Department of Psychology, Stanford University, Stanford, California 94305, ${ }^{2} \mathrm{Wu}$ Tsai Neurosciences Institute, Stanford University, Stanford, \\ California 94305, ${ }^{3}$ BCBL. Basque Center on Cognition, Brain and Language, Donostia-San Sebastián, Gipuzkoa 20009, Spain, and ${ }^{4}$ Department of \\ Psychology and Center for Neural Science, New York University, New York, New York 10003
}

The visual field region where a stimulus evokes a neural response is called the receptive field (RF). Analytical tools combined with functional MRI (fMRI) can estimate the RF of the population of neurons within a voxel. Circular population RF (pRF) methods accurately specify the central position of the $\mathrm{pRF}$ and provide some information about the spatial extent (diameter) of the RF. A number of investigators developed methods to further estimate the shape of the pRF, for example, whether the shape is more circular or elliptical. There is a report that there are many pRFs with highly elliptical pRFs in early visual cortex (V1-V3; Silson et al., 2018). Large aspect ratios $(>2)$ are difficult to reconcile with the spatial scale of orientation columns or visual field map properties in early visual cortex. We started to replicate the experiments and found that the software used in the publication does not accurately estimate RF shape: it produces elliptical fits to circular ground-truth data. We analyzed an independent data set with a different software package that was validated over a specific range of measurement conditions, to show that in early visual cortex the aspect ratios are $<2$. Furthermore, current empirical and theoretical methods do not have enough precision to discriminate ellipses with aspect ratios of 1.5 from circles. Through simulation we identify methods for improving sensitivity that may estimate ellipses with smaller aspect ratios. The results we present are quantitatively consistent with prior assessments using other methodologies.

Key words: visual cortex; population receptive field; fMRI

\section{Significance Statement}

We evaluated whether the shape of many population receptive fields (RFs) in early visual cortex is elliptical and differs substantially from circular. We evaluated two tools for estimating elliptical models of the pRF; one tool was valid over the measured compliance range. Using the validated tool, we found no evidence that confidently rejects circular fits to the pRF in visual field maps V1, V2, and V3. The new measurements and analyses are consistent with prior theoretical and experimental assessments in the literature.

\section{Introduction}

Small regions of the primate visual cortex (V1-V3) contain neurons whose spatial receptive fields (RFs) are compact and often overlap in the visual field. The RFs of individual neurons can be measured from electrical activity (Hubel and Wiesel, 1968). Using functional MRI (fMRI) responses, it is possible to measure the RFs of individual cortical voxels (Dumoulin and Wandell,

\footnotetext{
Received Dec. 3, 2020; revised Jan. 3, 2021; accepted Jan. 10, 2021.

Author contributions: G.L.-U., J.W., and B.A.W. designed research; G.L.-U., J.W., and B.A.W. performed research; G.L.-U. analyzed data; G.L.-U., J.W., and B.A.W. wrote the paper.

This work was supported by the European Union's Horizon 2020 Research and Innovation Program under the Marie Sklodowska-Curie Grant 795807 (to G.L.-U.) and by National Institutes of Health Grants EY027401, EY027964, and MH111417 (to J.W.). We thank E. Silson, C. Baker, and R. Reynolds. We also thank R. Reynolds for help with the AFNI software.

The authors declare no competing financial interests.

Correspondence should be addressed to Garikoitz Lerma-Usabiaga at garikoitz@gmail.com.

https://doi.org/10.1523/JNEUROSCI.3052-20.2021

Copyright $\odot 2021$ the authors
}

2008). These fMRI responses reflect the activity of many $\left(\sim 10^{5}\right)$ neurons and are called the population RF (pRF). There has been extensive work using pRF methods to measure visual cortex in the living human brain (Wandell and Winawer, 2015) and versions of these methods with intrinsic and calcium imaging have been used in animal model systems (Kalatsky and Stryker, 2003; Nauhaus et al., 2016).

The pRF estimates depend on models of the physiological response. The early $\mathrm{pRF}$ models used simple linear models of the physiological response, often assuming that the pRF has a canonical spatial profile (e.g., circularly symmetric Gaussian; Dumoulin and Wandell, 2008). Such simple models can accurately predict the fMRI time series of voxels in early visual cortex (e.g., V1-V3) when using a limited range of stimuli, capturing a very large proportion of the explainable variance. Over time, investigators have expanded the scope of the stimuli and this required increasing the complexity of the pRF models (Zuiderbaan et al., 2012; Kay et al., 2013; Greene et al., 2014; Alvarez et al., 2015). 
Table 1. Main parameters of the experiments

\begin{tabular}{|c|c|c|c|c|c|c|c|}
\hline Type & Simulated or real TR (s) & Duration (s) & Noise & Aspect ratio & Eccentricity $\left(^{\circ}\right)$ & pRF size $\left(^{\circ}\right)$ & pRF-analysis $^{*}$ \\
\hline \multirow[t]{3}{*}{ Synthetic } & 2 & 400 & None & $\{1,2\}$ & $\{1,2,3,4,5,6,7,8,9\}$ & $\{0.5,1,1.5,2,3,4\}$ & $\{$ AFNI6, Vista6\} \\
\hline & 2 & 400 & $\{l o w$, mid $\}$ & $\{1,2\}$ & $\{1,2,3,4,5,6,7,8,9\}$ & $\{0.25: 0.25: 6\}$ & $\{$ AFNI6, Vista6\} \\
\hline & 1 & $\{300400\}$ & $\{l o w, m i d\}$ & $\{1,2\}$ & $\{1,2,3,4,5,6,7,8,9\}$ & $\{0.25: 0.25: 6\}$ & $\{$ Vista6\} \\
\hline
\end{tabular}

* Note: 6 refers to the elliptical fits with six parameters, and 4 to the circular fits with four parameters.

This journal published a provocative claim that prior investigators had missed an important aspect of the human pRF shapes in V1-V3 (Silson et al., 2018). Nearly all the prior work in which a parametric form was assumed treated the pRF spatial profile as approximately circular. This question had been tested, for example, by Zeidman et al. (2018), who found that elliptical fits are not better than circular models. On the other hand, Silson et al. (2018) report that pRFs are significantly elongated, often with an aspect ratio (ratio of long to short axis) of 2.5 or greater. Groups using a broader class of allowable shapes have reported inconclusive results, for example, finding most pRFs in V1-V3 to be nearly circular but a small percentage to be quite elongated (Greene et al., 2014), or finding many voxels to be slightly elongated (Merkel et al., 2018, 2020).

We set out to investigate the discrepancy between the high ellipticity report and the more common assumption of near circularity by replicating the findings. We began the replication by using the same software as in Silson et al. (2018). As part of our workflow, we ran the software through a recently developed validation framework (Lerma-Usabiaga et al., 2020). This assessment revealed that the software returns inaccurate estimates, including ellipses with aspect ratios larger than 2 when tested with groundtruth circular data (aspect ratio of 1). To pursue the key scientific question, we decided to use a different software tool and to perform a full assessment of how accurately this tool might measure deviations from circularity. The validation of the second software tool identified a range of conditions where performance is reliable. Using the validated software with retinotopy data from the 7T Human Connectome Project, we find no support for a shape that is substantially and systematically different from circular.

\section{Materials and Methods}

For software validation we used synthetic data generated using the validation framework (pRF-synthesis) described by Lerma-Usabiaga et al. (2020). These methods are described in detail in that paper and summarized briefly here. We added two levels of noise (low-noise and midnoise) created with realistic models of several noise sources, including physiological noise (cardiac and respiratory), low frequency drift, and instrumental noise (white noise) derived from experimental measurements. We used bars with contrast patterns that swept the $20^{\circ}$ diameter visual field vertically, horizontally and in $45^{\circ}$ and $-45^{\circ}$, in two different directions each (eight-bar sweeps in total). The total stimulus duration and TR (sampling rate) were varied across several simulations (for details, see Table 1).

For assessing deviations from circularity of pRFs, we used empirical measurements from the 7T HCP Retinotopy project (Benson et al., 2018). Specifically, we selected retinotopy data collected from the three representative subjects analyzed in Benson et al. (2018; their Figure 7; HCP IDs 164131, 115017, and 536647). We analyzed the empirical data using the containers (pRF-analyze) described, implemented, and shared by Lerma-Usabiaga et al. (2020).

Experimental design and statistical analyses

We evaluated the mrVista (https:/github.com/vistalab/vistasoft) and AFNI (Cox, 1996) estimates of elliptical pRFs. The latter was introduced in Silson et al. (2018). The former is part of the mrVista toolbox but has not previously been used in published work.

In mid-2018, the AFNI development team discovered an error in the ellipse formula. Silson et al. (2018) re-ran the analyses and reported some numerical differences but no changes to the pattern of results or the conclusions: the pRF solutions remained highly elongated after correcting the code. Here, we used the new, corrected version of the software. The April fourth, 2020 version is implemented in the Docker container. The noiseless analyses in Figures 1,2 were performed in a local macOS binary installation with the August 28, 2018 version of AFNI. We validated the corrected algorithms using synthetic (groundtruth) input data and estimated the following parameters.

- The center position of the pRF $(x, y)$.

- The SDs $(\sigma 1-\sigma 2)$ of the two axes of the ellipse $(\sigma 1>\sigma 2$; circular fits are constrained to $\sigma 1=\sigma 2$ and one parameter is returned.)

- The angle $\theta$ of the main axis (larger $\sigma$ ). Not returned for the circular fit.

- The gain parameter $A$.

We estimated deviations from circular pRFs using the mrVista prf-Analyze container and measurements obtained from the HCP project. The pRF-Analyze-mrVista container returns the distribution of aspect ratio estimates $(\sigma 1 / \sigma 2)$ We compared median values and distributions from fitting the empirical measurements with values expected from analyzing ground-truth data generated using prfSynthesize.

Several analyses were performed in this manuscript using synthetic or real data (see Table 1 for the main parameters of the experiments).

\section{Code availability and reproducibility}

To reproduce the computations in this paper requires that MATLAB and Docker be installed on your computer. The configuration files and the HCP data for the empirical analyses are curated and stored in a project at the Open Science Foundation (OSF; https://osf.io/ $9 \mathrm{jhcm} /$ ). The software we describe downloads the data from that OSF project.

The code specific to this paper is shared in the GitHub repository PRFmodel that is within the vistalab project (https://github.com/ vistalab/PRFmodel.git). After cloning that repository, please select the git tag EllipsePaperv02. Place this repository on your MATLAB path. The script pmMainEllipseFiguresScript.m describes how to install the necessary support libraries and execute the relevant scripts.

The software for the pRF-validation framework (Lerma-Usabiaga et al., 2020), including the code used to synthesize the BOLD time series, is shared in the same repository. The Docker container image can be downloaded from Docker hub with the command docker pull garikoitz/ prfsynth. The mrVista analysis code is publicly shared in https://github. com/vistalab/vistasoft, and its container can be downloaded from Docker hub with the command docker pull garikoitz/prfanalyze-vista. The AFNI analysis code is publicly shared in https:/github.com/afni/ afni, and our containerized version used for the analyses in this paper can be downloaded from Docker hub with the command docker pull garikoitz/prfanalyze-afni.

\section{Results}

We present results about algorithm validity in noise-free and simulated noise conditions. We then define a range of 


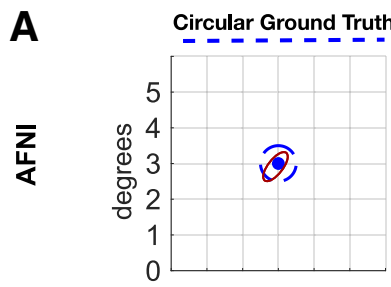

Fitted values
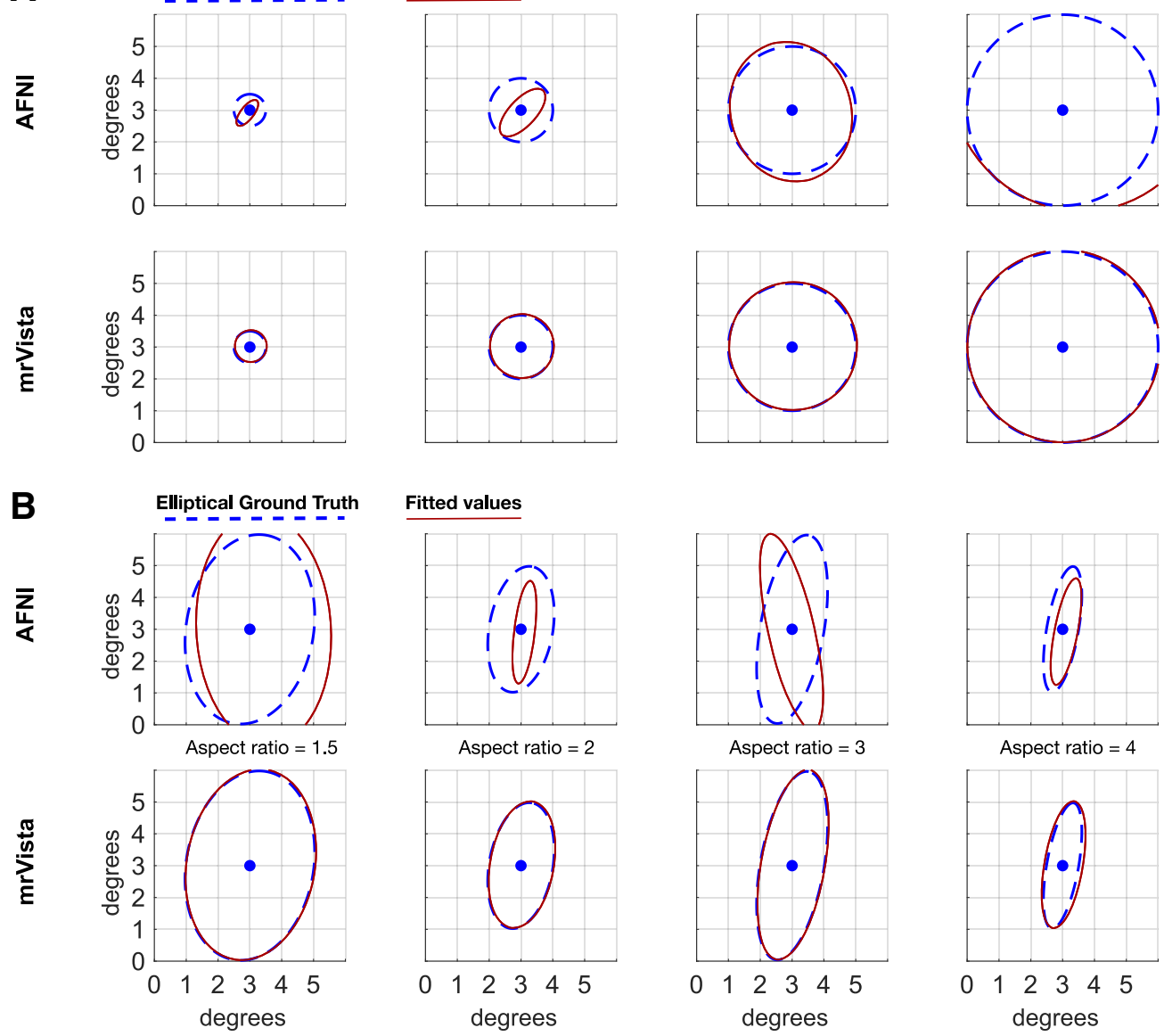

Figure 1. AFNI-elliptical does not recover accurate parameters of noise-free synthetic data. We analyzed noise-free synthetic data analysis with AFNI-elliptical and mrVista-elliptical. $\boldsymbol{A}$, AFNI-elliptical (top row) and mrVista-elliptical (bottom row) analyses of circular, Gaussian, ground-truth data with four different pRF sizes. The dashed line represents the 1 SD radius of the Gaussian. $\boldsymbol{B}$, Same as $\boldsymbol{A}$ but with elliptical ground truth data.
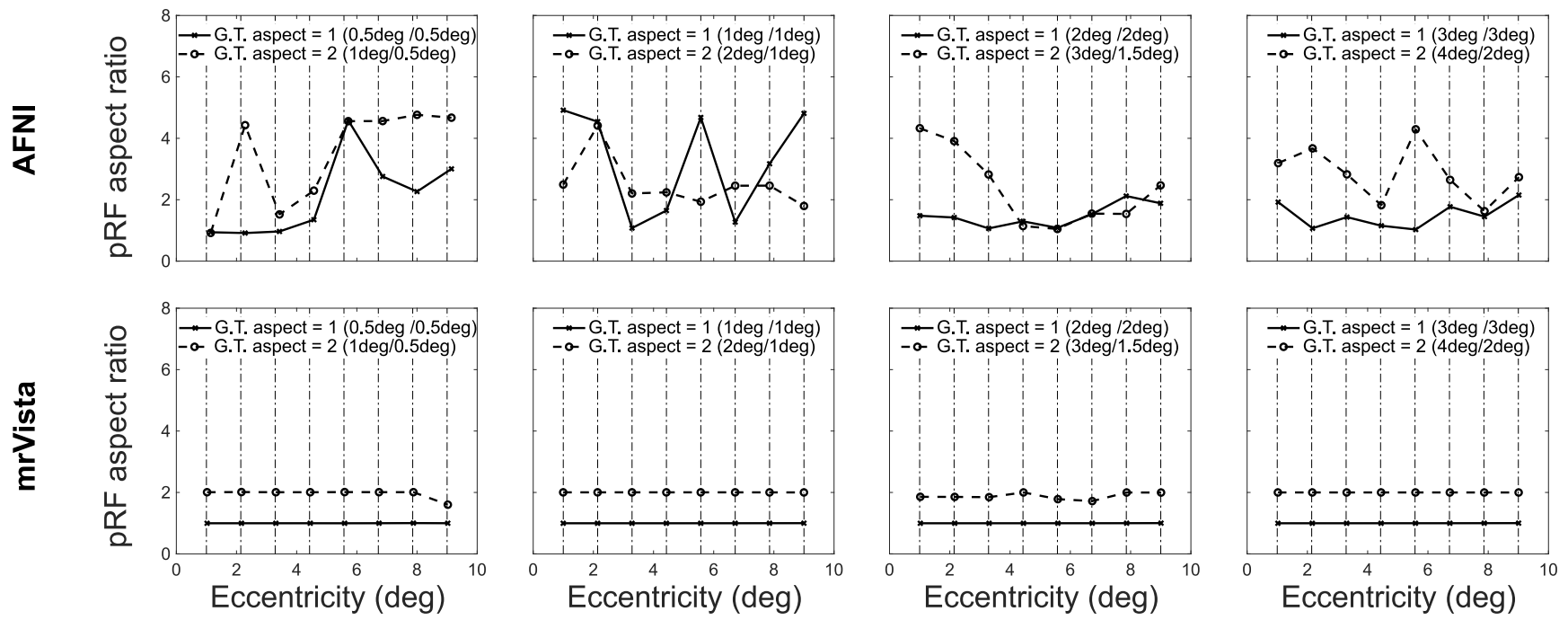

Figure 2. AFNI-elliptical has systematic aspect ratio errors at different eccentricities and sizes. AFNl-elliptical (top row) and mrVista (bottom row) results for circular (aspect ratio 1 , solid lines) and elliptical (aspect ratio 2, dashed lines) ground truth synthetic time series, with pRF radii ranging from $0.5^{\circ}$ to $4^{\circ}$. G.T., ground truth.

parameters in which one algorithm performs acceptably, and we analyze empirical measurements from that range. In previous work we reported that $\mathrm{pRF}$ algorithms systematically misestimate pRF parameters if there is a mismatch between the hemodynamic response function (HRF) used to simulate the time series with the HRF assumed in the analysis tool. Throughout the simulations here, we used synthetic data that matched the expected HRF. 


\section{AFNI-Elliptical}

A

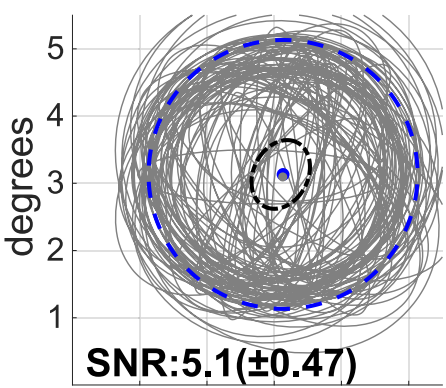

B

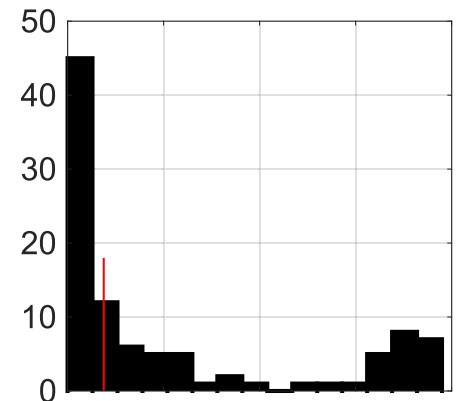

\section{mrVista-Elliptical}

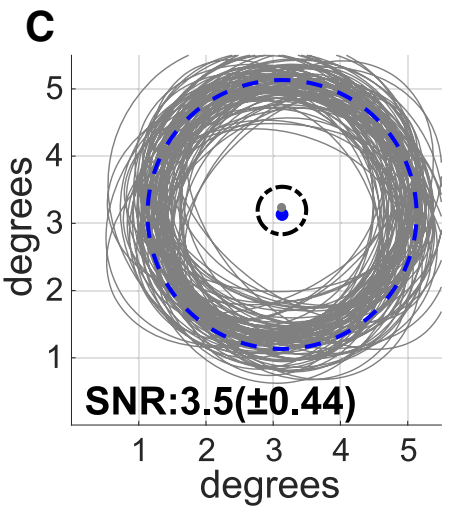

D

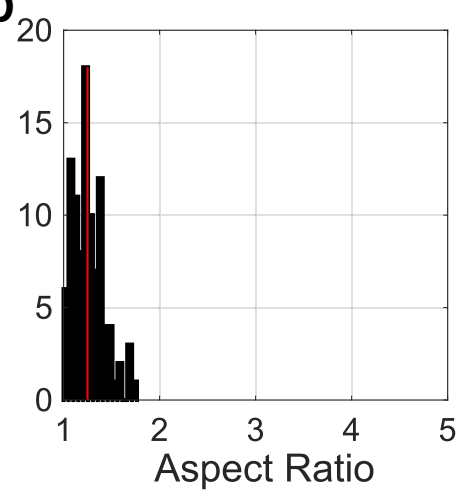

Figure 3. AFNl-elliptical estimates include large aspect ratios for circular ground-truth data with added noise. AFNI elliptical $(\boldsymbol{A}-\boldsymbol{B})$ and mrVista $(\boldsymbol{C}-\boldsymbol{D})$ analysis results for 100 noisy simulations (low noise). $\boldsymbol{A}-\boldsymbol{C}$, the representation of all the RFs (gray) over the ground truth (blue dashed line); the black dashed line contains the center locations and the blue dashed line represents the 1 SD radius of the Gaussian. $\boldsymbol{B}-\boldsymbol{D}$, the histogram of the aspect ratios. The median is indicated by the red line. SNR is the mean and STD of all 100 bold time series. Because of differences in the HRFs between the two algorithms and randomization used in the synthesis, the average SNR of the simulated time series differs, being lower for mrVista.

\section{Algorithm validity: noise-free analyses}

We first set out to validate elliptical models in AFNI ("AFNI-elliptical") and mrVista ("mrVista-elliptical") using noise-free synthetic data. We synthesized the BOLD time series for pRFs that are circular and centered at $(3,3)^{\circ}$, with radii spanning $0.5-3^{\circ}$. For these conditions, AFNI-elliptical inaccurately estimates the pRFs as elongated rather than circular, whereas mrVista-elliptical estimates nearly circular pRFs. We then validated the two algorithms with elliptical ground truth data (Fig. $1 B$ ). The groundtruth pRFs were again centered at $(3,3)^{\circ}$ and had aspect ratios between 1.5 and 4. Again, AFNI-elliptical fails to estimate the parameters accurately and mrVista-elliptical succeeds.

To explore whether there are systematic errors in AFNI-elliptical or mrVista-elliptical, we synthesized a noise-free dataset by systematically varying eccentricity, size, and aspect ratio (Fig. 2). The AFNI-elliptical algorithm generally returns incorrect aspect ratios. Over these parameter ranges, the mrVista-elliptical algorithm generally returns accurate estimates.

\footnotetext{
Algorithm robustness: noise analyses

In the presence of measurement noise, the aspect ratio of circular pRFs will be overestimated. Suppose that the ground truth is a circle with radius $\mathrm{r}$. The major and minor axes will both be estimates of the true radius plus noise, $r+\tilde{N}$. The estimated aspect ratio, $\mathrm{A}$, is the ratio of the two noisy samples constrained so that the major axis is the larger of the two samples:
}

$$
A=\max (r+\bar{N} 1, r+\bar{N} 2)) / \min (r+\bar{N} 1, r+\bar{N} 2) .
$$

From this formula, we observe that (1) the estimated value must be $>1$, and (2) the impact of the noise will be large when the pRF radius is small. We performed numerical simulations of the formula in Equation 1, using a range of radii and plausible noise distributions. We observed that for small radii starting at $0.25^{\circ}$ the median aspect ratio is of 2.5. The median aspect ratio values reduce asymptotically toward the aspect ratio of 1 as the radius increases.

We tested AFNI-elliptical and mrVista-elliptical using simulated noisy datasets for a circular $\mathrm{pRF}$ with a radius of $2^{\circ}$ (Fig. 3 ). The simulated stimulus had a TR $=2$, bar width of $2.8^{\circ}$ and step size of $1.2^{\circ}$; the simulated duration was $400 \mathrm{~s}$, including eightbar sweeps across the visual field. The time series were identical for 100 simulations except for different random samples of noise. The AFNI-elliptical algorithm estimates the center location accurately, but it does not estimate the aspect ratio as expected. There are many large aspect ratios $(>4)$, and there are many estimates of 1.0 , which should be rare given the noise. The mrVista-elliptical algorithm estimates the center location accurately. The median aspect ratio is generally in the range between 1.2 and 1.4, as expected. AFNI requires the aspect ratios to be bounded, so we restricted them to be between 1 and 5. MrVista has no such requirements, and therefore the aspect ratios were unbounded.

Based on the simulations, we expect the estimated aspect ratio of circular, noisy ground-truth data to be slightly larger than 1 . The mrVista-elliptical estimates conform to this expectation: they are distributed compactly around an aspect ratio of $1.26 \pm 0.16$ (Fig. 3D). The AFNI-elliptical estimates (Fig. $3 B$ ) are very different, with a larger mean and a much larger standard deviation: $2.07 \pm 1.36$. Critically, AFNI returns many aspect ratio estimates $>3$, which suggests that such values should not be taken as evidence of large aspect ratios in the data. A paired $t$ test comparing the magnitude of the aspect ratios showed that AFNI-elliptical's error is significantly bigger than mrVista-elliptical's $(t=6.7, p=$ 1.4e-09)

To test the generality of these findings, we synthesized and analyzed ground truth datasets with a broader range of parameters. For AFNI-elliptical (Fig. 4), the estimated aspect ratio for circular pRFs was $\sim 2.5-3.0$ for all ground truth radii (Fig. $4 A$ ). The distribution of values is quite wide, spanning all the aspect ratios within the $[1,5]$ bounds (Fig. $4 B$ ). When the ground-truth aspect ratio was 2 , the estimated aspect ratio increased to a median value of 4 , but the distribution remained very broad. These simulations used the mid-level of noise (see Materials and Methods). The results were similar for the low-level noise, and various eccentricity values. These validation tests reveal that, with our configuration, environment variables and function calls, AFNI-elliptical is not a suitable tool for assessing the aspect ratio of pRFs. The validation tests produce a wide range of aspect ratio distributions, as found in the empirical analyses reported by Silson et al. (2018). The reason for the differences in these AFNI distributions is unknown. 

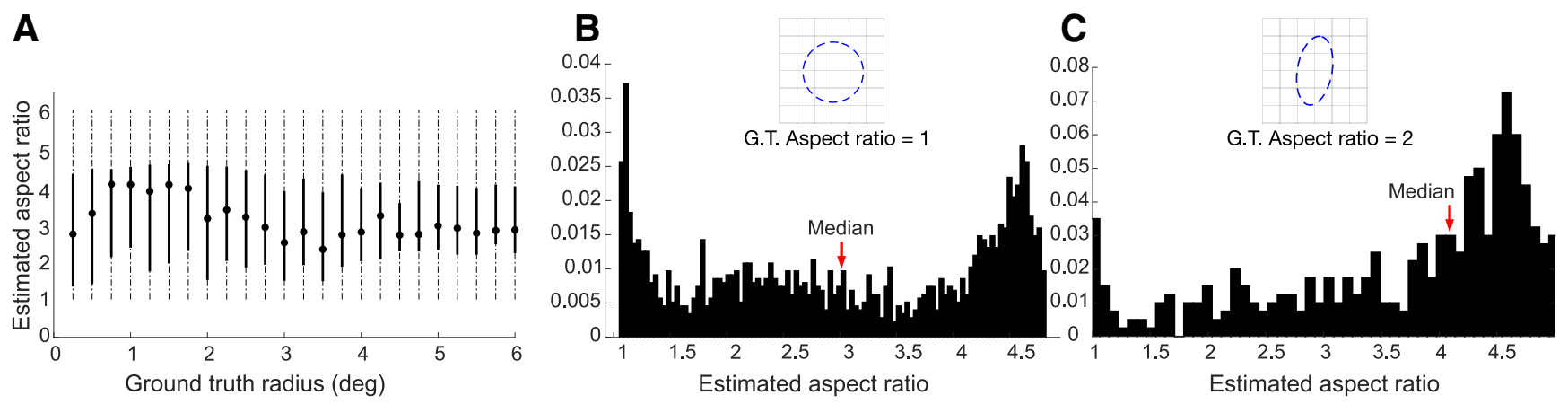

Figure 4. AFNI-elliptical does not estimate the correct aspect ratio of synthetic data. $A$, Estimated aspect ratio (ground truth aspect ratio $=1$ ) as a function of pRF radius $\left({ }^{\circ}\right.$ ). The points are the median and the lines show the range corresponding to the central $50 \%$ of the estimates. $\boldsymbol{B}$, Histogram of estimated aspect ratios (ground truth aspect ratio $=1$ ) using simulated pRFs with a mixture of radius sizes $\left(1-4^{\circ}\right)$ and eccentricities $\left(2-6^{\circ}\right)$. C, Histogram of estimated aspect ratios (ground truth aspect ratio $=2$ ) for the same mixture of radius sizes and eccentricities. The simulated bar width is $2.8^{\circ}$ and the bar translates $1.2^{\circ}$ for each TR $(2 \mathrm{~s})$. The simulations used the mid-level of noise. The red arrow indicates the median value of the histogram. G.T., ground truth.

$T R=2 s$.
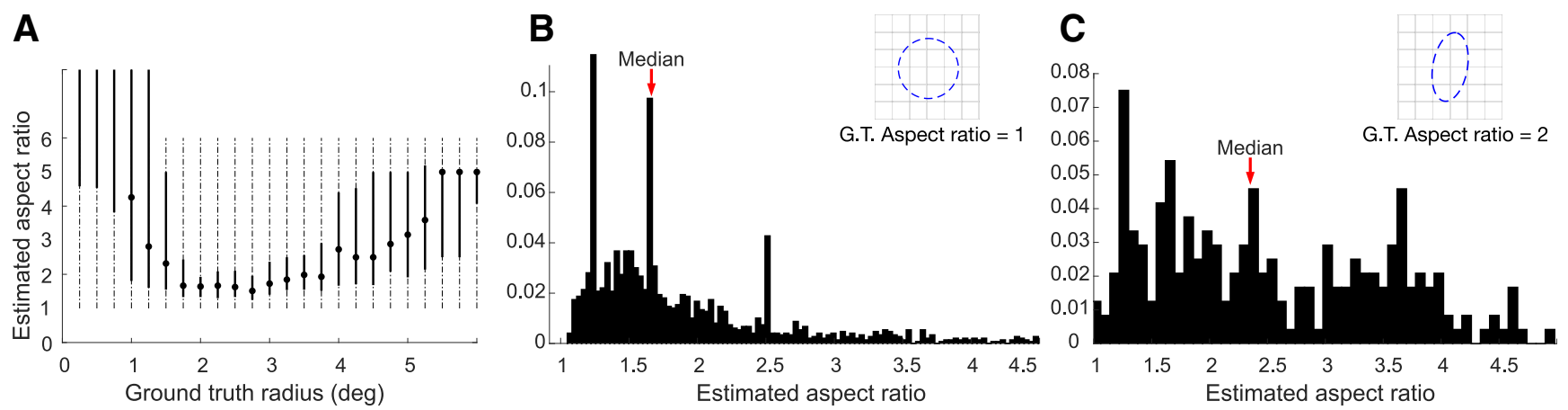

TR $=1 \mathrm{~s}$.
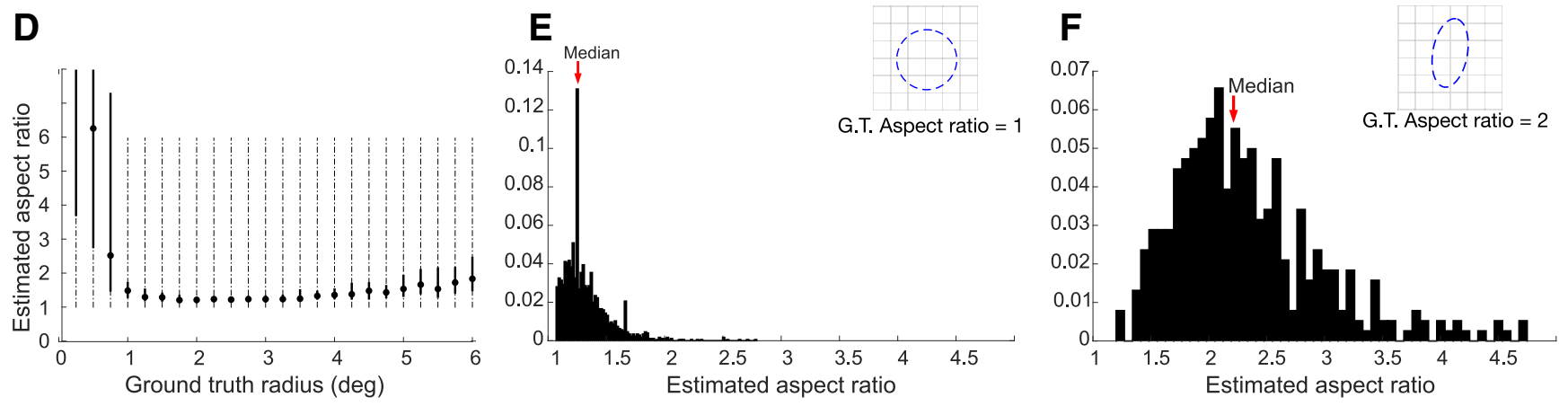

Figure 5. mrVista-elliptical aspect ratio estimates are close to accurate over a limited range of conditions. $A$, Estimated aspect ratio (ground truth $=1$ ) as a function of pRF radius $\left({ }^{\circ}\right.$ ). The points are the median and the lines show the range corresponding to $50 \%$ of the estimates. $\boldsymbol{B}$, Histogram of estimated aspect ratios (ground truth $=1$ ) using simulated pRFs with a mixture of radius sizes $\left(1-4^{\circ}\right)$ and eccentricities $\left(2-6^{\circ}\right)$. C, Histogram of estimated aspect ratios (ground truth $=2$ ) for the same mixture of radius sizes and eccentricities. The simulated bar width is $2.8^{\circ}$ and the bar translates $1.2^{\circ}$ for each TR $(2 \mathrm{~s})$. The simulations used the mid-level of noise. $\boldsymbol{D}-\boldsymbol{F}$, The same graphs calculated with a smaller bar displacement $\left(0.6^{\circ}\right)$ and shorter TR $(1 \mathrm{~s})$. For the large bar step size the mrVista elliptical estimates differ between the ground-truth aspect ratios of 1 and 2, although there is very poor accuracy when the ground truth aspect ratio is 2 . Reducing the bar step size and increasing the number of temporal samples improves the accuracy of the aspect ratio estimate (D-F). The peaks in the histogram at aspect ratios of $1.25,1.6$, and 2.5 in $\boldsymbol{B}, \boldsymbol{E}$ are a flaw in the algorithm. These peaks, which are present in fits to empirical data (below), are likely because of the coarse-to-fine search method implemented in the algorithm. These simulations define a range of experimental parameters where mrVista-elliptical provides useful information about the aspect ratio. G.T., ground truth.

We next analyzed the ability of mrVista-elliptical to accurately estimate the $\mathrm{pRF}$ aspect ratio (Fig. 5). As expected from the basic analysis of signal-to-noise (Equation 1), the accuracy of the aspect ratio estimates depends on pRF size and properties of the stimulus. With a TR of $2 \mathrm{~s}$, simulations with small $\mathrm{pRF}$ radius $\left(\sim 1^{\circ}\right.$ or less) are very inaccurate, including many large aspect ratios (Fig. $5 \mathrm{~A}$ ). We simulated the accuracy of recovering a circular pRF using a mixture of
pRF sizes $\left(1-4^{\circ}\right)$ and eccentricities $\left(2-6^{\circ}\right)$. The median estimated aspect ratio is $\sim 1.5$, with the estimates falling mostly between 1 and 2 (Fig. 5B). Simulating with a ground-truth aspect ratio of 2 increases the median, but the estimates are spread over a large range (Fig. 5C).

To understand how empirical methods using mrVista might impact algorithm validity, we conducted simulations with different experimental parameters. Specifically, we simulated an 
experimental protocol with a shorter TR ( $1 \mathrm{~s}$ instead of $2 \mathrm{~s}$ ), corresponding to a smaller stimulus step size. The mrVista-elliptical estimates are more accurate over a larger range (Fig. 5D). Estimating ground-truth pRFs with a range of sizes and eccentricities, the median aspect ratio is 1.25 and the range is more compact (Fig. 5E). The estimates for a ground truth aspect ratio of 2 and multiple pRF sizes have a median aspect ratio slightly larger than 2 (2.2). The same analysis performed with a 2-s TR and low noise results in estimates similar to the 1-s TR simulations.

The mrVista-elliptical algorithm has a numerical estimation error that biases the results to return certain aspect ratios (1.25, $1.65,2.5)$; these are the peaks in the histogram. We suspect this failure arises from the multi-resolution (coarse to fine) search methodology. The coarse fit uses a grid of parameter values, and the values of the aspect ratio in the grid include these three values. This limitation of the algorithm does not render it unusable for further exploration with real measurements under certain conditions.

\section{Empirical measurement: estimated aspect ratio}

We used mrVista-elliptical and experimental data to assess the aspect ratio of pRFs in early visual cortex. We analyzed three typical subjects from the HCP 7T retinotopy dataset (Benson et al., 2018). We first used mrVista-circular to assess the parameter ranges. The estimated range of eccentricity values $\left(2.5-6.5^{\circ}\right)$ and the pRF areas $\left(6.5-30^{\circ 2}\right)$ were then used to restrict the mrVistaelliptical fits. These parameters are consistent with many previous estimates and place no restriction on the estimated aspect ratio values. The HCP dataset was acquired with a TR of $1 \mathrm{~s}$ and a duration of $300 \mathrm{~s}$, and our analysis of synthetic data above indicates that in this spatial step, eccentricity and size range, the median mrVista-elliptical aspect ratios are reliable. Each subject had two runs with sweeping bar stimuli, and we analyzed the average of these runs.

We also created synthetic datasets with low-noise and midnoise levels to compare with the experimental data (Fig. 6). The synthetic datasets used the same sequence of stimulus apertures as the experimental data.

The analyses of the experimental data returned a median $\mathrm{pRF}$ aspect ratio of $\sim 1.5$, with no systematic effects of ventral versus dorsal, or eccentricity (Fig. 6A). The values are slightly larger in $\mathrm{V} 1$. There is no significant trend in the aspect ratio as a function of eccentricity. The aspect ratio from the experimental data are similar to the ratio in the mid-noise synthetic data. The experimental data aspect-ratios have higher variance than the synthetic values, but they are within the $95 \%$ confidence interval of the mid-noise synthetic aspect ratio estimations. As expected, the low-noise synthetic data simulations have aspect ratios closer to the ground truth value. This indicates that the mid-noise level of the synthetic data is a good approximation to the experimental data (Fig. 6B). When comparing experimental data from all maps, the histograms are similar, with median aspect ratio values at around 1.5. The validation procedures (Fig. 5) show that mrVista can accurately capture aspect ratios of 2 , and yet the empirical data return a distribution of aspect ratios that is smaller, closer to the theoretically expected values of a circular ground truth.

Finally, we analyzed the strength of the evidence in favor of using an elliptical model compared with the circular model. The elliptical model uses two more parameters and contains the circular model as a special case. Hence, it is expected that the variance explained $\left(R^{2}\right)$ will be higher for the elliptical model. In
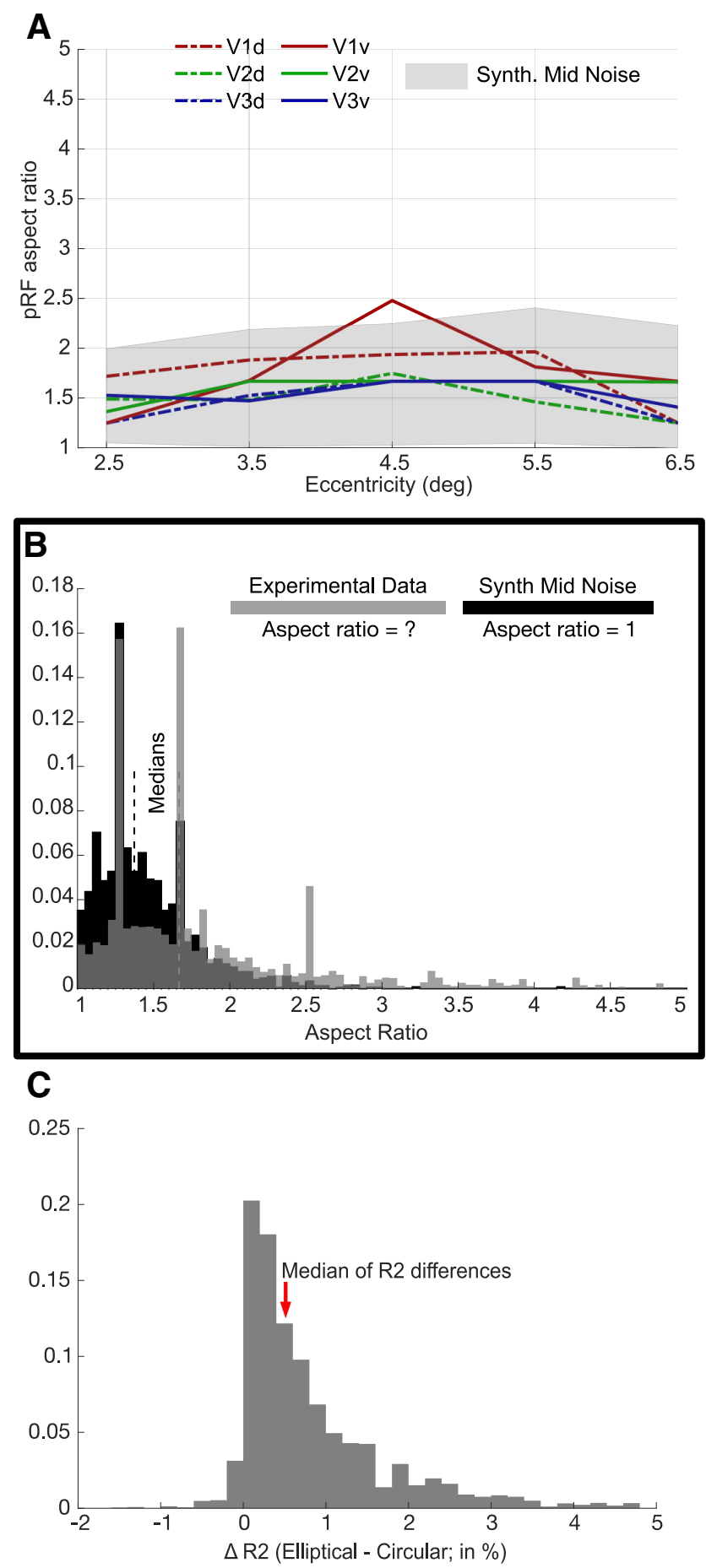

Figure 6. mrVista-elliptical pRF parameters estimated from empirical measurements in V1V3 $(N=3)$. A, Estimated median pRF aspect ratios of experimental (color) data plotted as a function of eccentricity. The experimental data are plotted separately for ventral and dorsal regions of V1-V3. Synthetic data were created using mid-level noise and are represented as a light gray band containing the central $95 \%$ aspect ratio values. The experimental data aspectratio fits show a large variance across voxels, but except one case, the population medians are within the expected range of the (mid) noise simulations. $\boldsymbol{B}$, Histograms of the estimated pRF aspect ratio for experimental (gray) and synthetic (black) data. Estimates were included in the histograms if the model fit explained at least $25 \%$ of the variance and the PRF position was between $2.5^{\circ}$ and $6.5^{\circ}$ and the PRF area size estimate was between $6.5^{\circ}$ and $30^{\circ 2}$. The ground truth aspect ratio for the synthetic data were 1. The thin dashed vertical lines represent the median values for the experimental and synthetic analyses. $C$, Histogram of the difference in variance explained $\left(R^{2}\right)$ between the elliptical and circular model fits to the experimental data. The histogram includes data subject to the same restrictions as in $\boldsymbol{B}$. The precise parameters for determining the restriction do not impact the conclusions in either $\boldsymbol{B}$ or $\boldsymbol{C}$. 
rare cases, the $R^{2}$ is lower for the elliptical model, indicating a failure of the optimization to find the best solution. Figure $6 C$ shows the histogram of the difference between the $R^{2}$ of the elliptical and circular fits, for all experimental data in which the models explain $>25 \%$ of the variance. The elliptical fit is systematically higher than the circular fit, but the median difference is $<1 \%$, and even the few voxels with the largest difference are no more than $5 \%$. Hence, there is almost no evidence in support of using the elliptical model over the circular model for these experimental data. Detecting differences from circularity will require new protocols and models.

Many analyses of these types of histograms, separating the data in various ways such as dorsal and ventral or by visual field map, support the same conclusions. The median aspect ratio values remain between 1.25 and 1.5 , and we found no systematic relationship between the estimated aspect ratio or ellipse orientation and $\mathrm{pRF}$ position in the visual field.

\section{Discussion}

\section{The application of pRF methods}

Multiple groups have used pRF parameters as dependent variables to understand the effects of cortical plasticity, attention, and diagnostic tools for neurology and psychiatry (Wandell and Winawer, 2015). For example, pRF methods have been used to examine hypotheses about brain substrate changes, such as excitation-inhibition imbalances, that may be associated with neurologic, ophthalmologic and psychiatric diseases (Papanikolaou et al., 2014; Wandell and Winawer, 2015; Anderson et al., 2017; Dumoulin and Knapen, 2018). There are also opportunities to understand individual differences in the visual pathways that may impact performance in tasks that rely on vision, such as reading (Le et al., 2017) and face recognition (Witthoft et al., 2016). Establishing the precision of the parameter estimates obtained with current protocols and tools enables us to determine with more confidence whether an individual under study is within the distribution of typical subjects.

\section{Oriented pRFs}

What would be a plausible biological basis for pRFs with large aspect ratios? Many neurons in primary visual cortex have oriented RFs. For simple cells, the spatial envelope tends to be elongated along the axis of orientation tuning (De Valois et al., 1982; Ringach, 2002; Michel et al., 2013). The neurons are arranged in an orderly pattern such that the main orientation changes smoothly across the cortical surface. The RFs span many orientations over a $1 \mathrm{~mm}$ distance (Hubel and Wiesel, 1974). In typical 3T measurements, a single fMRI voxel aggregates the response over a millimeter or more and thus accumulates the metabolic response from neurons with many orientations. It would be quite surprising if the orientation of neuronal RFs could be observed robustly in fMRI measurements. Although there have been some claims to this effect (Kamitani and Tong, 2005; Sasaki et al., 2006; Freeman et al., 2011), it appears now that these biases are because of properties of the stimulus aperture rather than to orientation tuning (Carlson, 2014; Roth et al., 2018)

Alternatively, the pRF from a voxel could be elongated if the neural RFs center positions within a voxel had an asymmetric distribution. Such asymmetric distributions might occur if, for example, the cortical magnification differed systematically between the radial and tangential directions. The effect of neural RF distribution within a voxel on the shape of the $\mathrm{pRF}$ is, however, likely to be modest (Amano et al., 2009). Were the pRF measurements

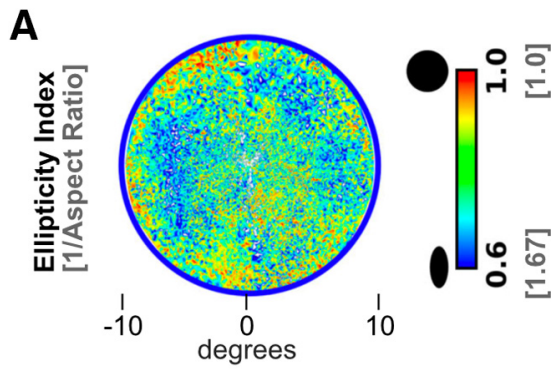

B

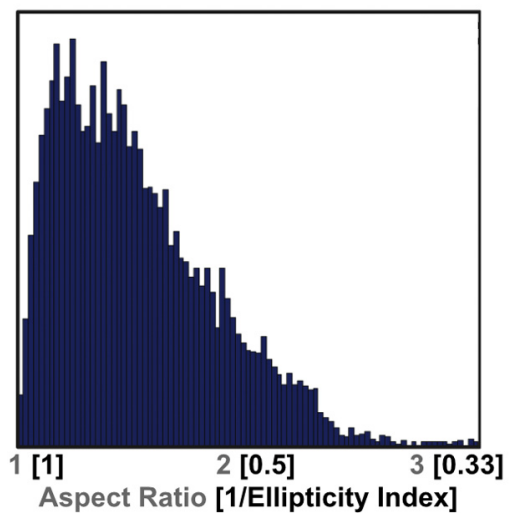

Figure 7. Ellipticity results reported in the literature show similar results to our circular ground truth simulations. $\boldsymbol{A}$, Ellipticity (1/aspect ratio) reported in Merkel et al. (2020). B Aspect ratio reported in Greene et al. (2014).

truly to have a large aspect ratio, we would still need to find a plausible biological basis.

We are unaware of claims other than Silson et al. (2018) that one can reliably measure a large aspect ratio based on the fMRI response from individual voxels. Direct comparisons of standard pRF models suggest that circular RF models provide the best fits (Zeidman et al., 2018; their Fig. 10). Using novel measurement approaches, investigators report that individual fMRI voxels may have some orientation preference with a magnitude similar to the values reported here (Greene et al., 2014; Merkel et al., 2018, 2020).

For example, Merkel et al. $(2018,2020)$ estimated the aspect ratios of voxels in early visual cortex and reported ellipticity (the inverse of aspect ratio) ranging between 0.6 and 1 , which corresponds to aspect ratios of 1-1.67 (Fig. 7A; Merkel et al., 2020). Using tomographic methods to estimate pRF shapes (Greene et al., 2014) also estimated aspect ratios (Fig. 7B). The distribution they report had $11 \%$ of the aspect ratios $>2$, which is close to the expected amount based on our simulations with synthetic data assuming circular pRFs (14\%) and based on analysis with the HCP 7T (8\%) data.

These aspect ratios are not meaningfully different from circular given the expected level of experimental noise and current protocols. Specifically, by definition the estimated aspect ratio value must exceed 1 . Further, the impact of experimental noise will be quite large when the pRF radius is small. For example, using $0.7^{\circ} \mathrm{SDs}$ as noise, for small radii $\left(0.25-1^{\circ}\right)$ the expected median aspect ratio is almost 3 . This value reduces asymptotically toward the aspect ratio of 1 for large $\mathrm{pRF}$ sizes $\left(>5^{\circ}\right)$. Moreover, $\mathrm{pRF}$ size estimates are less robust than $\mathrm{pRF}$ center estimates and the absolute value depends strongly on the individual HRFs (Lage-Castellanos et al., 2020; Lerma-Usabiaga et al., 2020). 
These principles and simulations show accurate estimation of aspect ratio values as small as 1.5 will require new experimental paradigms that mitigate instrumental noise and account for the computational uncertainties. It would also be preferable to use methods that include an accurate assessment of the individual subject's HRF. Elsewhere we used simulations to describe adjustments to experimental protocols that should improve the accuracy and stability of pRF measurements (Lerma-Usabiaga et al., 2020). Implementing and validating these methods will require some patience.

In conclusion, this project began with a report that the aspect ratios of pRFs in early visual cortex are substantially larger than previously thought (Silson et al., 2018). We set out to investigate this report, and we concluded that the difference could be traced to a software implementation. Our new data analysis confirmed the prior consensus about $\mathrm{pRF}$ shapes in early visual cortex: the best-fitting shapes are not very different from circular (Greene et al., 2014; Zeidman et al., 2018; Merkel et al., 2020). The ability to measure shapes with greater precision, perhaps revealing systematic deviations at individual voxels or even orientation maps, will require advances in protocols and analyses. Simulations suggest these may be in reach (Fig. 5).

The complexity of modern neuroimaging analyses has arrived at a point where explicit and public validation frameworks are important for building trust in publications and as part of the standard for software distribution. Here, we used the validation framework implemented in Lerma-Usabiaga et al. (2020). The development of validated models and quantified parameter estimates has been a hallmark of sensory science, and we continue that approach here.

\section{References}

Alvarez I, de Haas B, Clark CA, Rees G, Schwarzkopf DS (2015) Comparing different stimulus configurations for population receptive field mapping in human fMRI. Front Hum Neurosci 9:96.

Amano K, Wandell BA, Dumoulin SO (2009) Visual field maps, population receptive field sizes, and visual field coverage in the human MT+ complex. J Neurophysiol 102:2704-2718.

Anderson EJ, Tibber MS, Sam Schwarzkopf D, Shergill SS, Fernandez-Egea E, Rees G, Dakin SC (2017) Visual population receptive fields in people with schizophrenia have reduced inhibitory surrounds. J Neurosci 37:1546-1556.

Benson NC, Jamison KW, Arcaro MJ, Vu AT, Glasser MF, Coalson TS, Van Essen DC, Yacoub E, Ugurbil K, Winawer J, Kay K (2018) The Human Connectome Project 7 Tesla retinotopy dataset: description and population receptive field analysis. J Vis 18:23.

Carlson TA (2014) Orientation decoding in human visual cortex: new insights from an unbiased perspective. J Neurosci 34:8373-8383.

Cox RW (1996) AFNI: software for analysis and visualization of functional magnetic resonance neuroimages. Comput Biomed Res 29:162-173.

De Valois RL, Albrecht DG, Thorell LG (1982) Spatial frequency selectivity of cells in macaque visual cortex. Vision Res 22:545-559.

Dumoulin SO, Wandell BA (2008) Population receptive field estimates in human visual cortex. Neuroimage 39:647-660.

Dumoulin SO, Knapen T (2018) How visual cortical organization is altered by ophthalmologic and neurologic disorders. Annu Rev Vis Sci 4:357379.
Freeman J, Brouwer GJ, Heeger DJ, Merriam EP (2011) Orientation decoding depends on maps, not columns. J Neurosci 31:4792-4804.

Greene CA, Dumoulin SO, Harvey BM, Ress D (2014) Measurement of population receptive fields in human early visual cortex using back-projection tomography. J Vis 14:17.

Hubel DH, Wiesel TN (1968) Receptive fields and functional architecture of monkey striate cortex. J Physiol 195:215-243.

Hubel DH, Wiesel TN (1974) Uniformity of monkey striate cortex: a parallel relationship between field size, scatter, and magnification factor. J Comp Neurol 158:295-305.

Kalatsky VA, Stryker MP (2003) New paradigm for optical neurotechnique imaging: temporally encoded maps of intrinsic signal. Neuron 38:529545.

Kamitani Y, Tong F (2005) Decoding the visual and subjective contents of the human brain. Nat Neurosci 8:679-685.

Kay KN, Winawer J, Mezer A, Wandell BA (2013) Compressive spatial summation in human visual cortex. J Neurophysiol 110:481-494.

Lage-Castellanos A, Valente G, Senden M, De Martino F (2020) Investigating the reliability of population receptive field size estimates using fMRI. Front Neurosci 14:825.

Lerma-Usabiaga G, Benson N, Winawer J, Wandell BA (2020) A validation framework for neuroimaging software: the case of population receptive fields. PLoS Comput Biol 16:e1007924.

Le R, Witthoft N, Ben-Shachar M, Wandell B (2017) The field of view available to the ventral occipito-temporal reading circuitry. J Vis 17:6.

Merkel C, Hopf J, Schoenfeld MA (2018) Spatial elongation of population receptive field profiles revealed by model-free $f$ MRI back-projection. Hum Brain Mapp 39:2472-2481.

Merkel C, Hopf JM, Schoenfeld MA (2020) Modulating the global orientation bias of the visual system changes population receptive field elongations. Hum Brain Mapp 41:1765-1774.

Michel MM, Chen Y, Geisler WS, Seidemann E (2013) An illusion predicted by $\mathrm{V} 1$ population activity implicates cortical topography in shape perception. Nat Neurosci 16:1477-1483.

Nauhaus I, Nielsen KJ, Callaway EM (2016) Efficient receptive field tiling in primate V1. Neuron 91:893-904.

Papanikolaou A, Keliris GA, Papageorgiou TD, Shao Y, Krapp E, Papageorgiou E, Stingl K, Bruckmann A, Schiefer U, Logothetis NK, Smirnakis SM (2014) Population receptive field analysis of the primary visual cortex complements perimetry in patients with homonymous visual field defects. Proc Natl Acad Sci USA 111:E1656-E1665.

Ringach DL (2002) Spatial structure and symmetry of simple-cell receptive fields in macaque primary visual cortex. J Neurophysiol 88:455-463.

Roth Z, Heeger D, Merriam E (2018) Orientation selectivity and stimulus vignetting in human visual cortex. 2018 Conference on Cognitive Computational Neuroscience. Available at http://dx.doi.org/10.32470/ ccn.2018.1245-0.

Sasaki Y, Rajimehr R, Kim BW, Ekstrom LB, Vanduffel W, Tootell RBH (2006) The radial bias: a different slant on visual orientation sensitivity in human and nonhuman primates. Neuron 51:661-670.

Silson EH, Reynolds RC, Kravitz DJ, Baker CI (2018) Differential sampling of visual space in ventral and dorsal early visual cortex. J Neurosci 38:2294-2303.

Wandell BA, Winawer J (2015) Computational neuroimaging and population receptive fields. Trends Cogn Sci 19:349-357.

Witthoft N, Poltoratski S, Nguyen M, Golarai G, Liberman A, LaRocque KF, Smith ME, Grill-Spector K (2016) Reduced spatial integration in the ventral visual cortex underlies face recognition deficits in developmental prosopagnosia. bioRxiv. doi: 10.1101/051102.

Zeidman P, Silson EH, Schwarzkopf DS, Baker CI, Penny W (2018) Bayesian population receptive field modelling. Neuroimage 180:173-187.

Zuiderbaan W, Harvey BM, Dumoulin SO (2012) Modeling center-surround configurations in population receptive fields using fMRI. J Vis 12:10. 